\begin{tabular}{|c|c|}
\hline Title & Radiation feedback in dusty clouds \\
\hline Author(s) & Ishiki, Shohei; Okamoto, Takashi \\
\hline Citation & $\begin{array}{l}\text { Monthly notices of the royal astronomical society, 466(1), L123 L127 } \\
\text { https://doi.org/10.1093/mnrasl/slw253 }\end{array}$ \\
\hline Issue Date & 2017-03-21 \\
\hline Doc URL & http:/hdl.handle.net/2115/67327 \\
\hline Rights & $\begin{array}{l}\text { This article has been accepted for publication in Monthly Notices of the Royal A stronomical Society: Letters @: } 2016 \\
\text { Shohei I shiki, Takashi Okamoto Published by Oxford University Press on behalf of The Royal A stronomical Society. } \\
\text { A ll rights reserved. }\end{array}$ \\
\hline Type & article \\
\hline File Information & MNRA S466 L123-L127.pdf \\
\hline
\end{tabular}

Instructions for use 


\title{
Radiation feedback in dusty clouds
}

\author{
Shohei Ishiki ${ }^{\star}$ and Takashi Okamoto ${ }^{\star}$ \\ Department of Cosmoscience, Hokkaido University, N10 W8, Kitaku, Sapporo 060-0810, Japan
}

Accepted 2016 December 12. Received 2016 December 12; in original form 2016 December 1

\begin{abstract}
We have investigated the impact of photoionization and radiation pressure on a dusty starforming cloud using one-dimensional radiation hydrodynamic simulations, which include absorption and re-emission of photons by dust. We find that, in a cloud of mass $10^{5} \mathrm{M}_{\odot}$ and radius $17 \mathrm{pc}$, the effect of radiation pressure is negligible when star formation efficiency is 2 per cent. The importance of radiation pressure increases with increasing star formation efficiency or an increasing dust-to-gas mass ratio. The net effect of radiation feedback, however, becomes smaller with the increasing dust-to-gas mass ratio, since the absorption of ultraviolet photons by dust grains suppresses photoionization and hence photoheating.
\end{abstract}

Key words: radiative transfer-methods: numerical - ISM: clouds - H II regions.

\section{INTRODUCTION}

Radiative transfer is known to be very important in many astrophysical phenomena. Feedback from young, massive stars plays a crucial role in determining star formation activity and galaxy evolution. The energy and momentum input by stellar radiation from young, massive stars is most influential in a star-forming cloud before the explosion of the first supernova.

The radiation from young, massive stars can affect the surrounding medium through two channels. First, ultraviolet (UV) photons ionize the surrounding neutral gas and increase its temperature by photoheating. The $\mathrm{H}$ II region expands owing to the increased thermal pressure. This process disperses star-forming clouds (Walch et al. 2012) and changes star formation efficiency (Mac Low \& Klessen 2004; McKee \& Ostriker 2007). Secondly, neutral gas and dust absorb photons and acquire their momentum. The momentum pushes the material outwards. This process may drive galaxy scale outflows (Murray, Quataert \& Thompson 2005; Oppenheimer \& Davé 2006). The ionization feedback (Dale, Bonnell \& Whitworth 2007; Peters et al. 2010; Dale, Ercolano \& Bonnell 2012; Hosokawa et al. 2016) and radiation pressure feedback (Krumholz, Klein \& McKee 2007; Kuiper et al. 2010b, 2011, 2012; Kuiper \& Yorke 2013; Harries, Haworth \& Acreman 2014) are also important for individual star formation.

Recently, Sales et al. (2014) have studied these processes and have concluded that radiation pressure has negligible effect compared with photoheating. They, however, do not include dust in their simulations. On the other hand, Wise et al. (2012) perform radiation hydrodynamic simulations in full cosmological context and show that momentum input partially affect star formation by increasing

*E-mail: ishiki@astro1.sci.hokudai.ac.jp (SI); okamoto@astro1.sci. hokudai.ac.jp (TO) the turbulent support in early low-mass galaxies, while they ignore the dust since dust is unimportant in these low-metallicity systems.

The presence of dust increases the importance of radiation pressure because, unlike hydrogen and helium, dust can always absorb UV photons. Moreover, absorbed photons are re-radiated as infrared (IR) photons and again dust absorbs IR photons. Iterative process of the absorption and the re-emission increases the efficiency of converting photon energy to dust momentum. The creation of $\mathrm{H}$ II regions by UV radiation and momentum-driven gas outflows by absorption of re-emitted IR photons now become important ingredients in galaxy formation simulations, which are modelled as phenomenological subgrid physics (e.g. Hopkins, Quataert \& Murray 2011; Brook et al. 2012; Agertz et al. 2013; Stinson et al. 2013; Okamoto, Shimizu \& Yoshida 2014).

It is therefore important to investigate the radiative feedback by radiation hydrodynamic simulations that include re-emission from dust grains. To this end, we perform one-dimensional radiation hydrodynamic simulations in the presence of dust. In this Letter, we solve radiation transfer including angular dependence in order to deal with re-emission from dust and gas.

\section{METHODS}

We place a radiation source at the centre of a spherically symmetric gas distribution. To compare the effect of thermal and radiation pressure, we perform simulations with and without radiation pressure and investigate relative importance of these processes.

\subsection{Radiation transfer}

We here describe the algorithm that we use to solve the steady radiative transfer equation for a given frequency, $v$ :

$\frac{\mathrm{d} I_{v}}{\mathrm{~d} \tau_{v}}=-I_{v}+S_{v}$ 
where $I_{v}, \tau_{v}$, and $S_{v}$ are the specific intensity, the optical depth, and the source function, respectively. Optical depth of a ray segment, $\Delta \tau_{v}$, is determined as

$\Delta \tau_{v}=\kappa_{\nu} \Delta x=\sum_{i} n_{i} \sigma_{i} \Delta x$,

where $\kappa_{v}, n_{i}$, and $\sigma_{i}$ are the absorption coefficient, the number density, and the cross-section of $i$ th species of interest, respectively, and $\Delta x$ is the length of the ray segment that intersects the cell. The species we include in our simulations are $\mathrm{H}_{\mathrm{I}}, \mathrm{H}$ II, He I, He II, He III, electrons, and dust. We employ the cross-sections of $\mathrm{H}_{\mathrm{I}}, \mathrm{He} \mathrm{I}$, and He II given in Osterbrock (2006) and that of dust in Draine \& Lee (1984) and Laor \& Draine (1993). ${ }^{1}$

The recombination radiation from ionized hydrogen and helium is calculated as

$\frac{S_{\nu, i}}{\kappa_{v}}=\frac{\alpha_{\mathrm{i}}(T) n_{\mathrm{e}} n_{i} h v}{4 \pi \sqrt{\pi} \Delta v_{\mathrm{D}, i}} \mathrm{e}^{-\left(v-v_{0}\right)^{2} /\left(\Delta v_{\mathrm{D}}\right)^{2}}$,

where $h$ is the plank constant, $\alpha_{\mathrm{i}}$ is the recombination coefficient for a transition from ionized state to ground state, $v_{0, i}$ is the threshold frequency of the $i$ th species, $n_{\mathrm{e}}$ is the electron number density, $T$ is gas temperature, and $\Delta v_{\mathrm{D}, i}$ is the Doppler width defined as

$\Delta v_{\mathrm{D}, i}=\frac{v_{0}}{c} \sqrt{\frac{2 k T}{m_{i}}}$,

where $k$ is the Boltzmann constant and $c$ is the speed of light. For spherically symmetric systems, intensities are functions of radius and angle if the problem involves re-emission of photons as in our case. We therefore employ a scheme called the impact parameter method (Hummer \& Rybicki 1971).

\subsection{Chemical reactions and radiative heating and cooling}

In our simulations, we solve a network of chemistry consisting of $\mathrm{H}_{\mathrm{I}}, \mathrm{H}_{\text {II, }} \mathrm{He}_{\mathrm{I}}, \mathrm{He}$ II, He III, and electrons, which can be described by the following set of equations:

$\frac{\mathrm{d} n_{i}}{\mathrm{~d} t}=C_{i}-D_{i} n_{i}$,

where $n_{i}$ is number density of the $i$ th species, $C_{i}$ is the collective source term responsible for the creation of the $i$ th species, and the second term involving $D_{i}$ represents the destruction mechanisms for the $i$ th species. Since equation (5) is a stiff set of differential equations, we need an implicit scheme for solving them. We thus employ a backward difference formula (Anninos et al. 1997; Okamoto, Yoshikawa \& Umemura 2012):

$n_{i}^{t+\Delta t}=\frac{C_{i}^{t+\Delta t}+n_{i}^{t}}{1+D_{i}^{t+\Delta t} \Delta t}$,

where $C_{i}^{t+\Delta t}$ and $D_{i}^{t+\Delta t}$ are evaluated at the advanced timestep. Unfortunately, not all source terms can be evaluated at the advanced timestep due to the intrinsic non-linearity of equation (5). We thus sequentially update the number densities of all species in the order of increasing ionization states.

The chemical reactions included in our simulations are the recombination (Hummer 1994; Hummer \& Storey 1998), the collisional ionization (Janev et al. 1987; Abel et al. 1997), the dielectronic recombination (Aldrovandi \& Pequignot 1973), and the photoionization (Cen 1992).

\footnotetext{
${ }^{1}$ http://www.astro.princeton.edu/draine/dust/dust.diel.html
}

In order to determine the temperature of gas, we consider following radiative cooling and heating processes: the photoionization heating, the collisional ionization cooling, the dielectronic recombination cooling, the collisional excitation cooling (Cen 1992), the bremsstrahlung cooling (Hummer 1994), and the inverse Compton cooling (Ikeuchi \& Ostriker 1986), by assuming the cosmic microwave background radiation at $z=0$. Collisions between gas and dust grains and heating due to photoejection from grains (Yorke $\&$ Welz 1996) are not included in our simulations. We integrate the energy equation of gas implicitly as described in Okamoto et al. (2012).

\subsection{Dust}

We include absorption and thermal emission of photons by dust grains in our simulations. To convert mass density to number density, we assume a graphite grain whose size and density are $0.1 \mu \mathrm{m}$ and $1.0 \mathrm{~g} \mathrm{~cm}^{3}$, respectively, as a typical dust particle (Draine 2011). Dust temperature is determined by the radiative equilibrium, and thus the dust temperature is independent from gas temperature. We assume that the dust sublimation temperature is $1500 \mathrm{~K}$; however, dust could never be heated to this temperature in our simulations. We do not include photon scattering by dust grains for simplicity. Neglecting this process may overestimate the radiation pressure on dust grains, as we will discuss later.

\subsection{Timestepping}

We have to solve the static radiative transfer equation, the chemical reaction, and energy equations for gas and dust simultaneously. We thus iteratively solve these equations (Okamoto et al. 2012; Tanaka et al. 2015) until the relative difference in the electron number density, $n_{\mathrm{e}}$, and in the dust temperature, $T_{\mathrm{d}}$, in all cells become smaller than 0.5 per cent.

For this implicit time integration, we employ a timestep that is defined by the time-scale of the chemical reactions:

$\Delta t_{\text {chem }_{, k}}=0.1\left|\frac{n_{\mathrm{e}}}{\dot{n}_{\mathrm{e}}}\right|_{k}+1 \times 10^{-3}\left|\frac{n_{\mathrm{H}}}{\dot{n}_{\mathrm{H}}}\right|_{k}$,

where the subscript, $k$, denotes the cell number. The second term in the right-hand side prevents the timestep from becoming too short when the medium is almost neutral. We follow the evolution of the system with the minimum of the individual chemical timestep, $\Delta t_{\text {chem }}=\min \left(\Delta t_{\text {chem }, k}\right)$, if this timestep is shorter than a timestep defined by the Courant-Friedrichs-Lewy condition.

\subsection{Hydrodynamics}

Hydrodynamics is solved by using a scheme called AUSM+ (Liou 1996) in the second-order accuracy in space and time. In order to prevent cell density from becoming zero or a negative value, we set the minimum number density, $n_{\mathrm{H}} \simeq 10^{-10} \mathrm{~cm}^{-3}$. We have confirmed that our results are not sensitive to the choice of the threshold density as long as the threshold density is sufficiently low. Throughout this Letter, we assume that dust and gas are dynamically tightly coupled. We have performed test simulations described in Bisbas et al. (2015) and confirmed that our code reproduces their results. 


\subsection{Relative importance of radiation pressure and thermal} pressure

Krumholz \& Matzner (2009) introduce a parameter, $\zeta$, for quantifying the relative importance of radiation pressure and thermal pressure. The parameter is defined as

$\zeta=\frac{r_{\mathrm{ch}}}{r_{\mathrm{St}}}$

where $r_{\mathrm{ch}}$ is the radius at which the thermal pressure and the radiation pressure forces on an expanding shell are equal and $r_{\mathrm{St}}$ is the Strömgren radius calculated for the initial density distribution. For $\zeta>1$, the expansion becomes radiation-pressure dominated. In general, the value of $\zeta$ increases with luminosity of a radiation source and a dust-to-gas mass ratio. We estimate $\zeta$ for each simulation to compare our numerical results with the analytic predictions. To calculate $r_{\mathrm{ch}}$, we need to know how many times on average a photon is absorbed or scattered in a shell, $f_{\text {trap }}$ (Krumholz \& Matzner 2009). We estimate this value by an iterative procedure and obtain $f_{\text {trap }}=1$ for all our simulations.

\section{SIMULATION SETUP}

To study radiation feedback in star-forming clouds, we model each cloud as a Bonnor-Ebert sphere of mass $10^{5} \mathrm{M}_{\odot}$. The radius of a cloud is set to obey the Larson's law (Larson 1981). This gives the radius of a cloud to be $17 \mathrm{pc}$. As a radiation source, we place a simple stellar population (SSP) at the centre of the sphere. The SSP has the solar metallicity. We vary the mass of a radiation source for testing the role of source luminosity. We compute its luminosity and spectral-energy distribution as functions of time by using a population synthesis model, PÉGASE. 2 (Fioc \& RoccaVolmerange 1997, 1999), assuming the Salpeter initial mass function (Salpeter 1955). We use linearly spaced 128 meshes in radial direction, 128 meshes in angular direction, and 256 meshes in frequency direction to solve radiation hydrodynamics. We use reflective boundary conditions at the inner boundary and semipermeable boundary condition at the outer boundary.

Materials at radius, $r$, feel the radial gravitational acceleration,

$a_{\mathrm{g}}=-G \frac{M(<r)}{r^{2}}-G \frac{M_{\text {star }}}{\max \left(r^{3}, r_{\text {soft }}^{3}\right)} r$,

where $M(<r)$ represents the total mass of gas inside $r$ and $M_{\text {star }}$ is the mass of the central radiation source. The gravitational force due to the radiation source is softened for numerical stability by introducing the softening length $r_{\text {soft }}$, which is set to $0.5 \mathrm{pc}$.

To study the importance of dust in radiation feedback, we use five initial conditions, Clouds 1, 2, 3, X2, and X3, in which we vary the dust mass fraction and mass of the radiation source as follows. Cloud 1 is a dustless cloud, while Clouds 2 and X2 have the solar metallicity ${ }^{2}$ and we assume that half of the metals are in dust. Although Clouds 2 and X2 have a typical metallicity of starforming clouds, its IR optical depth from the cloud centre to the edge, $\tau_{\mathrm{IR}}=0.15\left(\sigma_{\mathrm{IR}}=2.3 \times 10^{-12} \mathrm{~cm}^{-2}\right)$, is much lower than the value of typical star-forming clouds ( $\tau_{\mathrm{IR}} \sim 1$; Agertz et al. 2013) because of the low central concentration of a Bonnor-Ebert sphere. We therefore apply a higher metallicity for Clouds 3 and X3 so that the IR optical depths of the clouds become unity. The initial mass of the radiation source is 2 per cent for Clouds 1,2 , and 3, while

${ }^{2}$ We employ the solar metallicity by Asplund et al. (2009).
20 per cent for Clouds X2 and X3. The details of initial conditions are listed in Table 1.

\section{RESULTS}

We present density, ionization fraction, dust temperature, and velocity profiles of each cloud in Fig. 1. In order to investigate the relative importance of each process, we perform simulations in which several physical processes are switched off. Simulations that include effect of increased thermal pressure due to photoheating are indicated by a label 'PH'. When simulations do not have this label, hydrogen and helium are transparent for photons (photoionization and photoheating are switched off). Simulations in which we consider radiation pressure are labelled 'RP'; in the simulations labelled 'RP', radiation pressure on hydrogen, helium, and dust is all included. Clouds 2, 3, X2, and X3 have dust, and thus simulations for these clouds have a label, 'Dust'. In some simulations that include dust, we ignore absorption of re-emitted photons from dust. The label 'DustR' indicates that dust can absorb re-emitted photons and hence multiple events of absorption and re-emission are enabled. Simulations that include dust and all radiative processes are named ' $\mathrm{PH}+\mathrm{RP}+$ Dust+DustR'.

By comparing simulations of Cloud 1, 'PH' and 'PH+RP', we confirm earlier results by Sales et al. (2014), that is, the effect of radiation pressure is negligible in dustless clouds. Radiation pressure is also negligible in Cloud 2 as expected from the value of $\zeta=0.54$. The shell expansion in this cloud is almost identical to that in Cloud 1. In Cloud 3 where the dust-to-gas mass ratio is increased, the effect of radiation pressure becomes visible.

To isolate the effect of radiation pressure on dust, we run simulations in which we ignore the photoheating (and photoionization) of hydrogen and helium (RP+Dust+DustR). By comparing 'PH+Dust+DustR' and 'RP+Dust+DustR' in Cloud 3, we find that thermal pressure plays a more important role than radiation pressure in Cloud 3 in spite of the large value of $\zeta$.

Adopting a higher star formation efficiency, i.e. higher source luminosity, also increases the relative importance of radiation pressure. In Clouds X2 and X3, radiation pressure is more important than in Clouds 2 and 3, respectively. In particular, thermal pressure is negligible in Cloud X3 compared with radiation pressure. Since we increase the mass of the radiation source, thermal pressure force alone cannot compete with the gravitational force (see $\mathrm{PH}+$ Dust+DustR); shell expansion is driven almost solely by radiation pressure in this case (see RP+Dust+DustR).

We then investigate the impact of absorption of reemitted photons by dust by comparing 'PH+RP+Dust' and ' $\mathrm{PH}+\mathrm{RP}+$ Dust+DustR'. We find that this effect is negligible in almost all clouds. Only in Cloud X3, radiation pressure is slightly enhanced by this process. Since Clouds 3 and X3 have the same IR optical depth, the importance of absorption of re-emitted IR photons should depend not only on the IR optical depth but also on the source luminosity.

For a given luminosity of a radiation source, a higher dust-to-gas mass ratio increases importance of radiation pressure. We, however, find that the net effect of radiation feedback (i.e. radiation pressure plus photoheating) is decreased by the increased dust-to-gas mass ratio; the shell radii in Clouds 1,2 , and 3 become smaller in the increasing order of the dust-to-gas mass ratio. The shell radius in Cloud X3 is also smaller than that in Cloud X2. Since the shell expansion in Cloud X3 is dominated by radiation pressure, radiation feedback might become stronger than in Cloud X2 by increasing the dust-to-gas mass ratio further. Doing that would enhance the 
Table 1. Initial conditions and numerical setup for simulations. The radius and the total mass of each cloud are indicated by $r_{\text {cloud }}$ and $M_{\text {total }}$. The number densities, $n_{\mathrm{H}}, n_{\mathrm{He}}$, and $n_{\mathrm{d}}$ indicate the initial number densities of hydrogen, helium, and dust in the innermost cell, respectively. The initial temperature of gas and dust are represented by $T_{\mathrm{g}}$ and $T_{\mathrm{d}}$, respectively. The mass of a central radiation source, which determines the luminosity, is indicated by $M_{\mathrm{star}}$. The dust optical depths from the centre to $r_{\text {cloud }}$ at $3.29 \times 10^{15}$ and $1.76 \times 10^{13} \mathrm{~Hz}$ are, respectively, shown as $\tau_{\mathrm{d}, \mathrm{UV}}$ and $\tau_{\mathrm{d}, \mathrm{IR}}$.

\begin{tabular}{|c|c|c|c|c|c|c|c|c|c|c|c|}
\hline Cloud & $\begin{array}{c}r_{\text {cloud }} \\
(\mathrm{pc})\end{array}$ & $\begin{array}{l}M_{\text {total }} \\
\left(\mathrm{M}_{\odot}\right)\end{array}$ & $\begin{array}{c}n_{\mathrm{H}} \\
\left(\mathrm{cm}^{-3}\right)\end{array}$ & $\begin{array}{c}n_{\mathrm{He}} \\
\left(\mathrm{cm}^{-3}\right)\end{array}$ & $\begin{array}{c}n_{\mathrm{d}} \\
\left(10^{-9} \mathrm{~cm}^{-3}\right)\end{array}$ & $\begin{array}{l}T_{\mathrm{g}} \\
(\mathrm{K})\end{array}$ & $\begin{array}{l}T_{\mathrm{d}} \\
(\mathrm{K})\end{array}$ & $\begin{array}{c}M_{\mathrm{star}} \\
\left(10^{3} \mathrm{M}_{\odot}\right)\end{array}$ & $\tau_{\mathrm{d}, \mathrm{UV}}$ & $\tau_{\mathrm{d}, \mathrm{IR}}$ & $\zeta$ \\
\hline Cloud 1 & 17 & $10^{5}$ & 796 & 67 & 0 & 1074 & 10 & 2 & 0 & 0 & 0.13 \\
\hline Cloud 3 & 17 & $10^{5}$ & 761 & 64 & 19 & 1134 & 10 & 2 & 146 & 1.0 & 4.5 \\
\hline Cloud X2 & 17 & $10^{5}$ & 791 & 67 & 2.9 & 1082 & 10 & 20 & 22 & 0.15 & 5.0 \\
\hline Cloud X3 & 17 & $10^{5}$ & 761 & 64 & 19 & 1134 & 10 & 20 & 146 & 1.0 & 43 \\
\hline
\end{tabular}

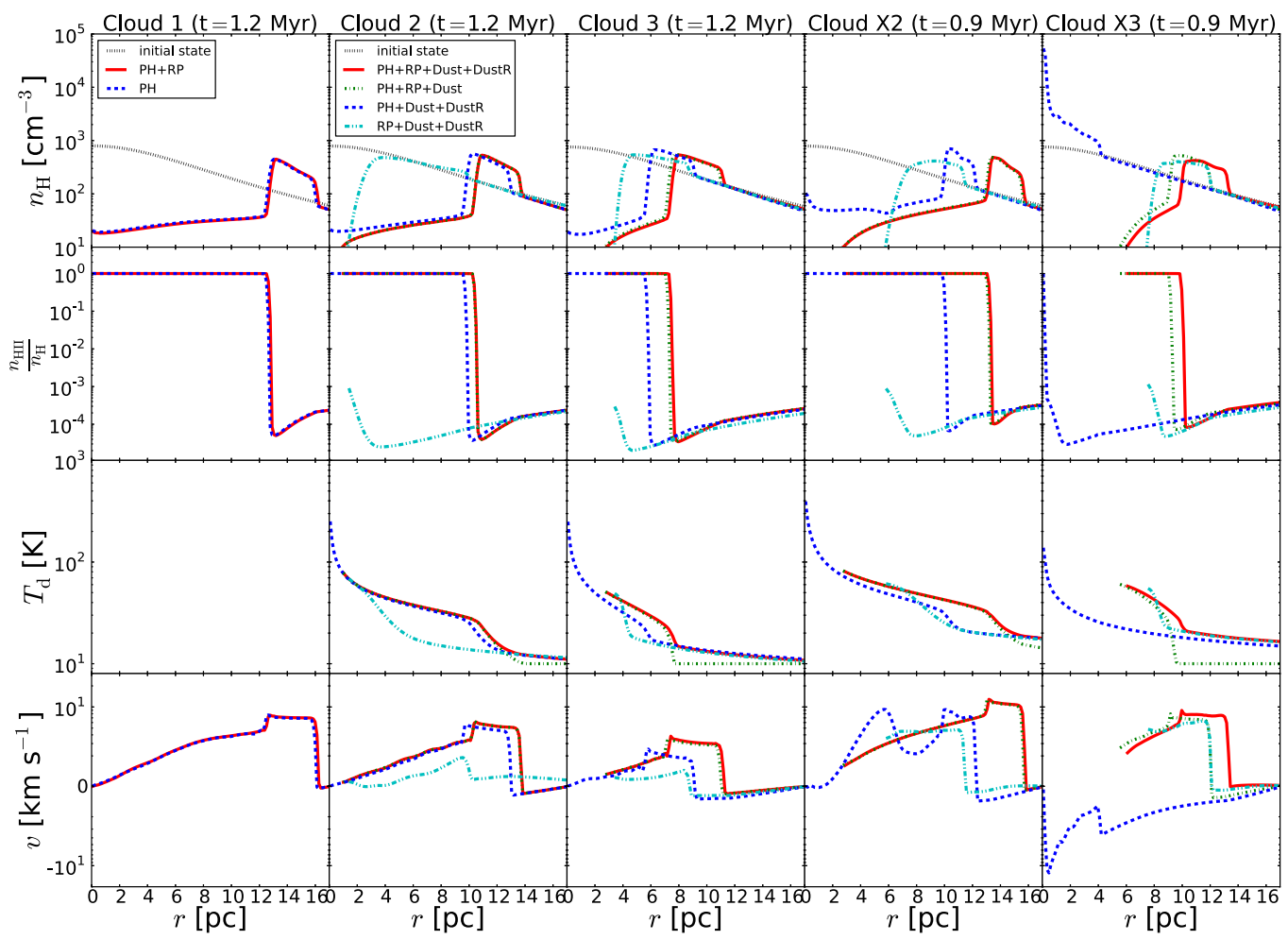

Figure 1. Density (top), ionization fraction (second from the top), dust temperature (second from the bottom), and velocity (bottom) profiles at $t=0.2 \mathrm{Myr}$. From left to right, we show the results for Clouds 1, 2, 3, X2, and X3. The black dotted lines in the top panels indicate the initial density profiles. The red solid lines represent the results of simulations that include all radiative transfer effects ('PH+RP' for Cloud 1 and 'PH+RP+Dust+DustR' for Clouds 2, 3, X2, and X3). The blue dashed lines show the results of simulations in which we ignore radiation pressure ('PH' for Cloud 1 and 'PH+Dust+DustR' for Clouds 2 , 3, X2, and X3). For Clouds 2, 3, X2, and X3, we perform simulations in which we include radiation pressure but we do not include absorption of re-emitted photons ('PH+RP+Dust'; green dot-dashed lines). Hydrogen and helium do not interact with photons in simulations labelled as 'RP+Dust+DustR', and hence photoheating is disabled in these simulations (cyan double-dot-dashed lines).

radiation pressure via multiple events of absorption of re-emitted photons. The adopted dust-to-gas mass ration for Cloud X3 is, however, already unrealistically high and, therefore, such a high dust-to-gas mass ratio would not be realized.

\section{DISCUSSION AND CONCLUSIONS}

We have investigated radiation feedback in dusty clouds of radius $17 \mathrm{pc}$ by one-dimensional radiation hydrodynamic simulations. In order to treat recombination radiation and re-emission from dust, we utilize the impact parameter method for radiation transfer.

We find that radiation pressure is negligible in a dustless cloud as pointed out by Sales et al. (2014). Radiation pressure is almost negligible when we adopt the solar metallicity and a low star formation efficiency ( 2 per cent: Cloud 2). This result seems to support the idea proposed by Krumholz \& Matzner (2009), that is, shell expansion is mainly driven by thermal pressure when the parameter, $\zeta$, is smaller than unity. By increasing a dust-to-gas mass ratio, the importance of radiation pressure is increased. Although values of $\zeta$ in Cloud 3, and X2 are significantly larger than unity, thermal pressure is still more dominant than radiation pressure in driving shell expansion. In all cases, radiation feedback creates a high density, neutral, expanding shell, which may trigger succeeding star formation (Hosokawa \& Inutsuka 2006).

We also find that effect of absorption of re-emitted photons is negligible in almost all clouds. Only in Cloud X3, radiation pressure 
is slightly enhanced by this process. We conclude that radiation pressure cannot be significantly boosted by this process on cloud scale unless either the star formation efficiency or the dust-to-gas mass ratio is extremely high.

In our simulations, radiation feedback becomes weaker for a given source luminosity as the dust-to-gas mass ratio increases by suppression of photoheating. This result is inconsistent with the assumption commonly made in cosmological simulations, that is, radiation feedback becomes stronger with the IR optical depth due to multiple events of absorption and re-emission of IR photons. (e.g. Hopkins et al. 2011; Agertz et al. 2013; Aumer et al. 2013; Okamoto et al. 2014). Our simulations are, however, on cloud scale and the IR optical depth is unity at maximum. Krumholz \& Matzner (2009) estimate $\zeta$ in starburst galaxies and they find, in some cases, $\zeta$ exceeds 1000 . For such a large value of $\zeta$, radiation energy would be efficiently converted into radiation pressure, and radiation feedback might become stronger for a larger IR optical depth. To test this, we have to perform radiation hydrodynamic simulations for starburst galaxies.

Our simulations likely overestimate the impact of radiation feedback by three reasons. First, we model a star-forming cloud as a Bonnor-Ebert sphere. In reality, however, star-forming clouds are highly turbulent and characterized by self-similar fractal structure (Falgarone, Phillips \& Walker 1991; Elmegreen \& Falgarone 1996; Stutzki et al. 1998). Photons preferentially escape through lowdensity medium due to the anisotropy of the thermal radiation field when a cloud has such complex density structure, and thus dust obtains less momentum compared with that in a spherically symmetric cloud (Kuiper et al. 2010b, 2011, 2012; Kuiper \& Yorke 2013). In order to properly deal with this situation, we should perform threedimensional radiative hydrodynamics simulations that include reemission from dust, which are currently computationally too expensive (but see Kuiper et al. 2010a). Secondly, we assume that gas and dust are tightly coupled. Although this assumption is commonly made (e.g. Netzer \& Elitzur 1993), dust would leave gas behind at the shock front because dust grains obtain large momentum from photons and create sharp shocks in our simulations. If this had happened, the net impact of radiation pressure on gas would become weaker than in our simulations. Finally, we do not include photon scattering by dust grains. In reality, grains are moderately reflective and strongly forward scattering in UV (see Draine 2003). The forward scattering of UV photons could strongly decrease the radiative pressure feedback. We defer these issues to future studies.

\section{ACKNOWLEDGEMENTS}

We thank the referee, Rolf Kuiper, for careful reading of our manuscript and for useful comments. We are grateful to Takashi Kozasa and Takashi Hosokawa for helpful discussion. We acknowledge the financial support of Japan Promotion of Science Grantin-Aid for Young Scientists (B:24740112) and MEXT KAKENHI Grant (16H01085).

\section{REFERENCES}

Abel T., Anninos P., Zhang Y., Norman M. L., 1997, New Astron., 2, 181 Agertz O., Kravtsov A. V., Leitner S. N., Gnedin N. Y., 2013, ApJ, 770, 25 Aldrovandi S. M. V., Pequignot D., 1973, A\&A, 25, 137

Anninos P., Zhang Y., Abel T., Norman M. L., 1997, New Astron., 2, 209

Asplund M., Grevesse N., Sauval A. J., Scott P., 2009, ARA\&A, 47, 481

Aumer M., White S. D. M., Naab T., Scannapieco C., 2013, MNRAS, 434, 3142
Bisbas T. G. et al., 2015, MNRAS, 453, 1324

Brook C. B., Stinson G., Gibson B. K., Wadsley J., Quinn T., 2012, MNRAS, 424, 1275

Cen R., 1992, ApJS, 78, 341

Dale J. E., Bonnell I. A., Whitworth A. P., 2007, MNRAS, 375, 1291

Dale J. E., Ercolano B., Bonnell I. A., 2012, MNRAS, 424, 377

Draine B. T., 2003, ARA\&A, 41, 241

Draine B. T., 2011, Physics of the Interstellar and Intergalactic Medium. Princeton Univ. Press, Princeton, NJ

Draine B. T., Lee H. M., 1984, ApJ, 285, 89

Elmegreen B. G., Falgarone E., 1996, ApJ, 471, 816

Falgarone E., Phillips T. G., Walker C. K., 1991, ApJ, 378, 186

Fioc M., Rocca-Volmerange B., 1997, A\&A, 326, 950

Fioc M., Rocca-Volmerange B., 1999, preprint (astro-ph/9912179)

Harries T. J., Haworth T. J., Acreman D. M., 2014, in Stamatellos D., Goodwin S., Ward-Thompson D., eds, Astrophysics and Space Science Proceedings, Vol. 36, The Labyrinth of Star Formation. Springer, Cham, Switzerland, p. 395

Hopkins P. F., Quataert E., Murray N., 2011, MNRAS, 417, 950

Hosokawa T., Inutsuka S.-i., 2006, ApJ, 646, 240

Hosokawa T., Hirano S., Kuiper R., Yorke H. W., Omukai K., Yoshida N., 2016, ApJ, 824, 119

Hummer D. G., 1994, MNRAS, 268, 109

Hummer D. G., Rybicki G. B., 1971, MNRAS, 152, 1

Hummer D. G., Storey P. J., 1998, MNRAS, 297, 1073

Ikeuchi S., Ostriker J. P., 1986, ApJ, 301, 522

Janev R. K., Langer W. D., Post D. E., Evans K., 1987, Elementary Processes in Hydrogen-Helium Plasmas. Springer-Verlag, Berlin

Krumholz M. R., Matzner C. D., 2009, ApJ, 703, 1352

Krumholz M. R., Klein R. I., McKee C. F., 2007, ApJ, 656, 959

Kuiper R., Yorke H. W., 2013, ApJ, 772, 61

Kuiper R., Klahr H., Dullemond C., Kley W., Henning T., 2010a, A\&A, 511, A81

Kuiper R., Klahr H., Beuther H., Henning T., 2010b, ApJ, 722, 1556

Kuiper R., Klahr H., Beuther H., Henning T., 2011, ApJ, 732, 20

Kuiper R., Klahr H., Beuther H., Henning T., 2012, A\&A, 537, A122

Laor A., Draine B. T., 1993, ApJ, 402, 441

Larson R. B., 1981, MNRAS, 194, 809

Liou M.-S., 1996, J. Comput. Phys., 129, 364

Mac Low M.-M., Klessen R. S., 2004, Rev. Mod. Phys., 76, 125

McKee C. F., Ostriker E. C., 2007, ARA\&A, 45, 565

Murray N., Quataert E., Thompson T. A., 2005, ApJ, 618, 569

Netzer N., Elitzur M., 1993, ApJ, 410, 701

Okamoto T., Yoshikawa K., Umemura M., 2012, MNRAS, 419, 2855

Okamoto T., Shimizu I., Yoshida N., 2014, PASJ, 66, 70

Oppenheimer B. D., Davé R., 2006, MNRAS, 373, 1265

Osterbrock D. E. F. G. J., 2006, Astrophysics of Gaseous Nebulae and Active Galactic Nuclei, 2nd edn. University Science Books, Mill Valley, CA

Peters T., Banerjee R., Klessen R. S., Mac Low M.-M., Galván-Madrid R., Keto E. R., 2010, ApJ, 711, 1017

Sales L. V., Marinacci F., Springel V., Petkova M., 2014, MNRAS, 439, 2990

Salpeter E. E., 1955, ApJ, 121, 161

Stinson G. S., Brook C., Macciò A. V., Wadsley J., Quinn T. R., Couchman H. M. P., 2013, MNRAS, 428, 129

Stutzki J., Bensch F., Heithausen A., Ossenkopf V., Zielinsky M., 1998, A\&A, 336, 697

Tanaka S., Yoshikawa K., Okamoto T., Hasegawa K., 2015, PASJ, 67, 62

Walch S. K., Whitworth A. P., Bisbas T., Wünsch R., Hubber D., 2012, MNRAS, 427, 625

Wise J. H., Abel T., Turk M. J., Norman M. L., Smith B. D., 2012, MNRAS, 427,311

Yorke H. W., Welz A., 1996, A\&A, 315, 555

This paper has been typeset from a $\mathrm{T}_{\mathrm{E}} \mathrm{X} / \mathrm{LT} \mathrm{E} \mathrm{X}$ file prepared by the author. 